\title{
Dynamics of Heart Rate and Sleep Stages in Normals and Patients with Sleep Apnea
}

\begin{abstract}
Thomas Penzel ${ }^{*, 1}$, Jan W Kantelhardt ${ }^{2,3}$, Chung-Chang Lo ${ }^{3}$, Karlheinz Voigt ${ }^{4}$ and Claus Vogelmeier'
'Division of Pulmonary Diseases, Department of Internal Medicine, Hospital of Philipps-University, Marburg, Germany; ${ }^{2}$ Institut für Theoretische Physik III, Justus-Liebig-Universität, Giessen, Germany; ${ }^{3}$ Center for Polymer Studies and Department of Physics, Boston University, Boston, USA; ${ }^{4}$ Institute for Physiology, Philipps-University, Marburg, Germany

Sleep is not just the absence of wakefulness but a regulated process with an important restorative function. Based on electroencephalographic recordings and characteristic patterns and waveforms we can distinguish wakefulness and five sleep stages grouped into light sleep, deep sleep, and rapid-eye-movement (REM) sleep. In order to explore the functions of sleep and sleep stages, we investigated the dynamics of sleep stages over the night and of heart-rate variability during the different sleep stages. Sleep stages and intermediate wake states have different distri butions of their duration and this allows us to create a model for the temporal sequence of sleep stages and wake states. Heart rate is easily accessed with a high precision by the recording and analysis of the electrocardiogram (ECG). Heart-rate regulation is part of the autonomous nervous system and sympathetic tone is strongly influenced by the sleep stages. Neuropsychopharmacology (2003) 28, S48-S53. doi:I0. I038/s..npp. I 300 I 46
\end{abstract}

Keywords: sleep; sleep stages; sleep apnea; heart-rate variability; nonlinear dynamics

\section{INTRODUCTION}

Sleep is not just the absence of wakefulness but has an own internal structure. The internal structure can be described by sleep stages that are differentiated based on typical patterns and waves found in the electroencephalography (EEG), electro-oculography (EOG), and electromyography (EMG) recordings. The technique of sleep recording and the definition of sleep stages has been given in recommendations compiled by Rechtschaffen and Kales (1968) and these are still regarded as the universal common language of sleep medicine. Sleep stages were defined as 'wake' with dominant beta and alpha waves, 'stage 1' with less alpha waves slow rolling eye movements, 'stage 2' with sleep spindles, K-complexes, some theta waves, some delta waves, 'stage 3' with up to $50 \%$ delta waves, 'stage 4' with more than 50\% delta waves, and low EMG amplitude. During 'rapid-eye-movement (REM) sleep' EMG amplitude drops to its lowest values and the EEG is wake like with possible sawtooth waves but no alpha waves. Characteristic for REM sleep are the REMs. These sleep stages are evaluated in consecutive 30-s epochs. Each 30-s epoch of the sleep recording is labeled with one sleep stage. This results in a

\footnotetext{
* Correspondence: Dr T Penzel, Division of Pulmonary Diseases, Department of Internal Medicine, Hospital of Philipps-University, Baldingerstrasse I, D-35033 Marburg, Germany, Tel: +49 642I 2866435, Fax: +4964212865450 ,

E-mail: penzel@mailer.uni-marburg.de

Received 01 September 2002; revised 03 November 2002; accepted 03 December 2002
}

sleep profile. In normal sleep, the stages follow a structured sequence starting with wake, then light sleep with stages 1 and 2, followed by deep sleep (slow wave sleep) with stages 3 and 4 , and then followed by REM sleep. Such a sequence is called a sleep cycle which has a typical duration of 90 $110 \mathrm{~min}$. A normal night consists of six sleep cycles where the proportion of deep sleep decreases from the beginning to the end of the night and the proportion of REM sleep increases at the same time.

Today sleep laboratories perform cardiorespiratory sleep studies (ATS, 1989), which adds the recording of respiration and further signals. Respiration is recorded with respiratory effort sensors at the chest and the abdomen, effective oronasal airflow with thermistor sensors or as recently discussed with nasal pressure transducers (Hosselet et al, 1998), snoring with a laryngeal microphone and oxygen saturation derived from a finger or ear oximetry. The electrocardiogram (ECG) is recorded routinely, EMG of both legs is recorded to detect movement disorders during sleep. Today's sleep laboratories use computer-based equipment that allows digital recording, evaluation, and archiving of the sleep records. The evaluation of sleep is still based on visual inspection of all traces since automatic analysis of sleep recordings still has many limitations not acceptable to the sleep physician (Penzel and Conradt, 2000).

\section{OBSTRUCTIVE SLEEP APNEA AS A MODEL SLEEP DISORDER}

Obstructive sleep apnea is a sleep disorder characterized by repetitive cessations of respiratory flow for at least $10 \mathrm{~s}$. 
Typically sleep apnea events last for 30-60 s. The respiratory cessations are owing to a collapse of the upper airways that are normally kept open during breathing. With the drop of muscular tone during the period of falling asleep in patients with sleep apnea there is a faster drop in muscular tone of the upper airways than the diaphragm and the accessory respiratory muscles. Thus with a continued muscular effort of breathing during sleep, the upper airways collapse when the negative intrathoracic pressure created for inspiration during each breath becomes too strong to keep the upper airways open. Each apnea ends with a central nervous activation (arousal), which reconstitutes the regulation of breathing. The arousal is caused by the drop in oxygen and the increase in carbon dioxide during the course of the apnea. These arousals remain below the level of wakefulness and are not noticed by the patient affected by sleep apnea. A patient with sleep apnea may have 600 single apnea events in one night. Not always the upper airway obstruction needs to be complete. A partial obstruction is called hypopnea. A patient suffers from sleep apnea if he has more than five apnea and hypopnea events per hour of sleep. This number is defined as the apnea-hypopnea index (AHI).

Cardiovascular changes accompany every single apnea event. With each apnea heart rate decreases. During the apnea we do see a relative bradycardia. After the end of the apnea, when respiration reconstitutes a relative tachycardia is observed. Blood pressure decreases during the apnea and increases near the end of the apnea as the sympathetic tone increases. During the few breaths enormous increases in blood pressure were observed. Oxygen saturation does drop with the cessation of respiration and reaches its nadir during the few restituting breaths. Swings in oxygen saturation and heart rate are so characteristic that they have been used for early detection of sleep apnea with portable diagnostic devices (Roos et al, 1993). The leading symptom in patients with sleep apnea is excessive daytime sleepiness. Excessive daytime sleepiness is a sign of severe sleep fragmentation that is caused by the repetitive arousal during sleep. Even if the arousal remains below the level of consciousness, the temporal sequence of sleep stages is severely disrupted and the restorative function of sleep is destroyed. Sleep apnea is regarded as an independent risk factor for systemic hypertension (Grote et al, 1999).

Sleep apnea can be treated easily by applying a simple physical method. A continuous positive airway pressure (CPAP) applied through the nose using a tight mask increases the air pressure inside the upper airways (Sullivan et al, 1981). Thus, the upper airways are prevented from collapsing when the muscular tone drops during the period of falling asleep. The apneas disappear, the cardiovascular changes disappear and the sleep fragmentation is reversed. Studies on the treatment effect of CPAP could prove a reversal of daytime sleepiness (Jenkins et al, 1999) and of arterial hypertension even during daytime (Pepperell et al, 2002). This pneumatic method has to be used continuously and regularly during each night with sleep. If the CPAP is not taken, the apnea reappears immediately and also the sleep fragmentation comes back quickly. Sleep apnea can be regarded as a model disorder for sleep fragmentation causing excessive daytime sleepiness (Figure 1). This is very useful when studying the temporal sequence of sleep stages.

\section{SLEEP STAGES DYNAMICS}

The evaluation of sleep results in a sleep profile as introduced earlier. The conventional description for clinical reporting is the calculation of the percent of time spent in a specific sleep stage. Normal values have been reported for these percentages. In summary, about $50-60 \%$ of time is spent in light sleep, $15-20 \%$ of time is spent in deep sleep, $20-25 \%$ is spent in REM sleep, and $5 \%$ or less is spent in wakefulness (Carscadon and Dement, 2000). The number of arousal from sleep and the number of wake periods is counted in order to give an impression on sleep fragmenta-

Sleep profile in a patient with sleep apnea

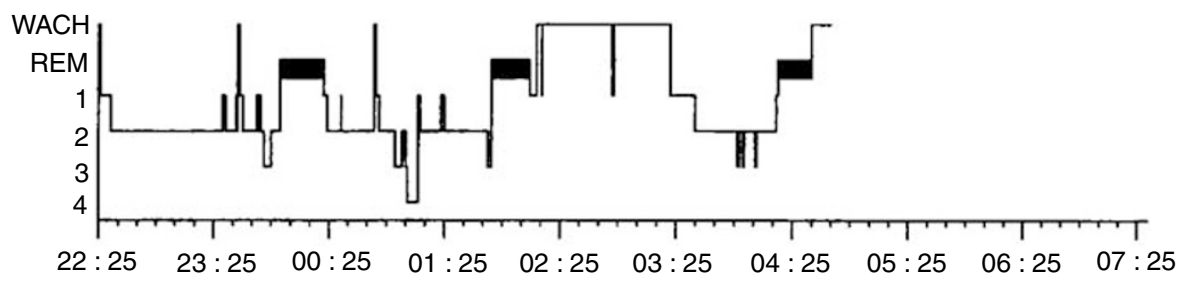

Sleep profile in the same patient under nCPAP therapy

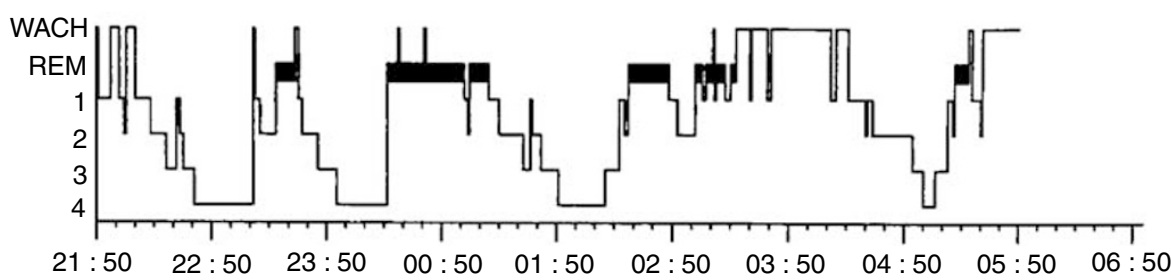

Figure I The sleep profile of a patient with severe sleep apnea shows a reduced percentage of deep sleep (stages 3 and 4), an increased percentage of light sleep (stages I and 2), and a long period of wakefulness. Under treatment with nasal CPAP that effectively removes all apnea events during the night, this patient had a very strong rebound effect on deep sleep. The high amount of deep sleep indicates a severe deep sleep deprivation. The patient also has a lot of REM sleep, which indicates some REM sleep deprivation. Still the patient has a long period of wakefulness, which is because of the effects of getting used to this new therapy. 
tion. Sometimes the number of transitions between sleep stages are also counted. Apart from these values there exist no normative data. Sleep fragmentation is often described in simple words but not given in quantitative numbers.

We wanted to derive a quantitative description that goes beyond the simple counting of sleep-stage transitions. In order to better characterize the dynamics of sleep-stage transitions, we produced statistics on the duration of the sleep stages. For our study, we chose data from a normative set of sleep recordings obtained in normal subjects and subjects with sleep apnea. These sleep recordings were evaluated by two independent sleep experts, which strictly followed the guidelines of Rechtschaffen and Kales. After that a third sleep expert reviewed the evaluations of the two experts. In the case the two experts obtained different classifications, the third expert had to decide which one to choose. In this manner, we arrived at the best possible sleep scoring for our subjects.

In order to obtain a new quantitative description we applied methods of statistical physics to the sleep profiles (Lo et al, 2002). The duration of the joined sleep stages (defined as light, deep, and REM sleep combined) followed an exponential distribution with a characteristic time scale $\tau$,

$$
p(t) \sim \exp (-t / \tau) \quad \text { with } \tau=22 \pm 1 \mathrm{~min} .
$$

In contrast, the duration of the short wake periods during sleep followed a completely different distribution (Figure 2). They showed a scale-free power-law distribution,

$$
p(t) \sim t^{-\alpha} \quad \text { with } \alpha=1.3 \pm 0.1 .
$$

In general, the value of the power-law exponent $\alpha$ remains the same when the time scale or time resolution changes. A power-law distribution is usually generated by complex dynamics, such as self-organized criticality (Sornette, 2000). It is very different from an exponential decay, which is usually generated by random processes in limited scale. For an introduction to scale-free power laws, see for example, Liebovitch (1998) and Bunde et al (2002, Chapter 8). In our analysis over the period of the night we did not find a measurable variation of the power-law distribution, which means that the value of $\alpha$ is constant. We found changes of the characteristic time scale of sleep periods throughout the night, which means that $\tau$ decreases from $27 \pm 1$ min in the beginning of the night to $18 \pm 1 \mathrm{~min}$ at the end of the sleep recording.

Based on these results we developed a stochastic random walk model with added forces pulling the walker towards sleep (Lo et al, 2002). The model shows a good agreement with the data. It suggests that the differences in the underlying laws for the dynamics of wake and sleep stages arise from the constraints on the number of stages in the sleep-wake system. We assumed that there are completely different neural systems involved in the regulation of sleep stages and wakefulness. We do not think that there is just a switching between wakefulness and sleep states in the same neurons, but different parts of the brain are involved. This speculation is the result of a statistical analysis of the observed sleep profiles and not of direct neuronal studies. To our surprise we did not find different laws for the distribution of REM sleep and non-REM sleep durations. This leads to the speculation that although both compo-
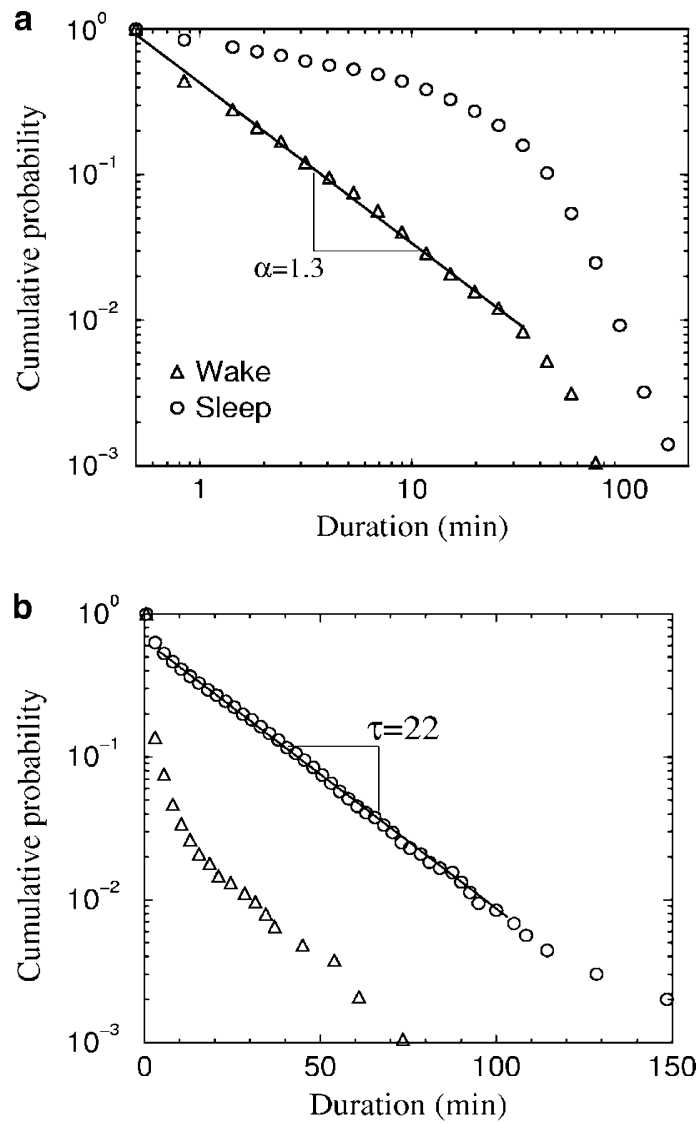

Figure 2 The distributions of the durations of wake and sleep phases are plotted in (a) double-logarithmic and (b) linear-logarithmic presentation. This makes the different underlying laws clear. Wake period durations follow a scale-free power-law distribution (a) characterized by the exponent $\alpha$. Sleep period durations follow an exponential distribution (b) characterized by the slope $\tau$. These sleep recordings from 197 subjects were obtained as part of the multicenter study SIESTA.

nents of sleep have completely different physiological expressions, both are still regulated in the same way by the integral sleep regulation.

When we investigated the distribution of the duration of sleep stages in patients with sleep apnea, we found the same laws. This confirms the universality of this regulation behavior in view of the fact that sleep apnea is a disorder that causes severe sleep fragmentation. Only the actual values of $\alpha$ and $\tau$ were different. We plan to use these differences for diagnostic purposes.

\section{DYNAMICS OF HEART-RATE VARIABILITY}

In order to find out how sleep and more particularly different sleep stages (that reflect different brain activity) affect the autonomous nervous system, we investigated one of its representative signals - heart rate. The recording of the ECG has become standard for sleep recordings. The current evaluation is limited to the simple statistics of heartrate variability and a rough estimate of nocturnal arrhythmias. A full diagnosis of arrhythmias requires the recording of more than one ECG lead as used in sleep recordings. The autonomous nervous system changes with sleep. Heart rate, 
blood pressure, and respiratory rate are lowered to adapt to the reduced metabolic needs during normal sleep. Consequently, the mean heart-rate values drop from wakefulness to light sleep and further to deep sleep. During REM sleep heart rate increases again showing a high variability, which may exceed the variability observed during quiet wakefulness (Zemaityte et al, 1986). Heart-rate variability has been investigated using spectral analysis in order to derive sympathetic and parasympathetic activity being attributed to specific frequency ranges (Akselrod et al, 1981). Using this analysis sleep stage specific changes of autonomic activities were identified (Zemaityte et al, 1986, Berlad et al, 1993). The spectral analysis had also been applied to sleep apnea subjects. One study showed that the characteristic pattern of bradycardia and tachycardia could be attributed to an effective parasympathetic control of heart rate interrupted by sympathetic activation at the apnea terminating arousal (Bonsignore et al, 1997).

We applied detrended fluctuation analysis (DFA) to detect the scaling behavior of interbeat interval time series in ECG recordings during sleep of 14 normal volunteers (Figure 3a) and 64 sleep apnea patients (Figure 3b) (Bunde et al, 2000, Penzel et al, 1999). The DFA method (see eg Kantelhardt et al, 2001; Bunde et al, 2002, Chapters 5, 7, and 8 ) has become a widely used technique for the determination of the scaling properties and the detection of longrange correlations in noisy time series, since it avoids a spurious detection of correlations that are artifacts of nonstationarities. It allows to distinguish real long-range correlations characterized by a power-law decay of the autocorrelation function from short-range correlations characterized by a finite decay time. Here, the method was separately applied to the data recorded during
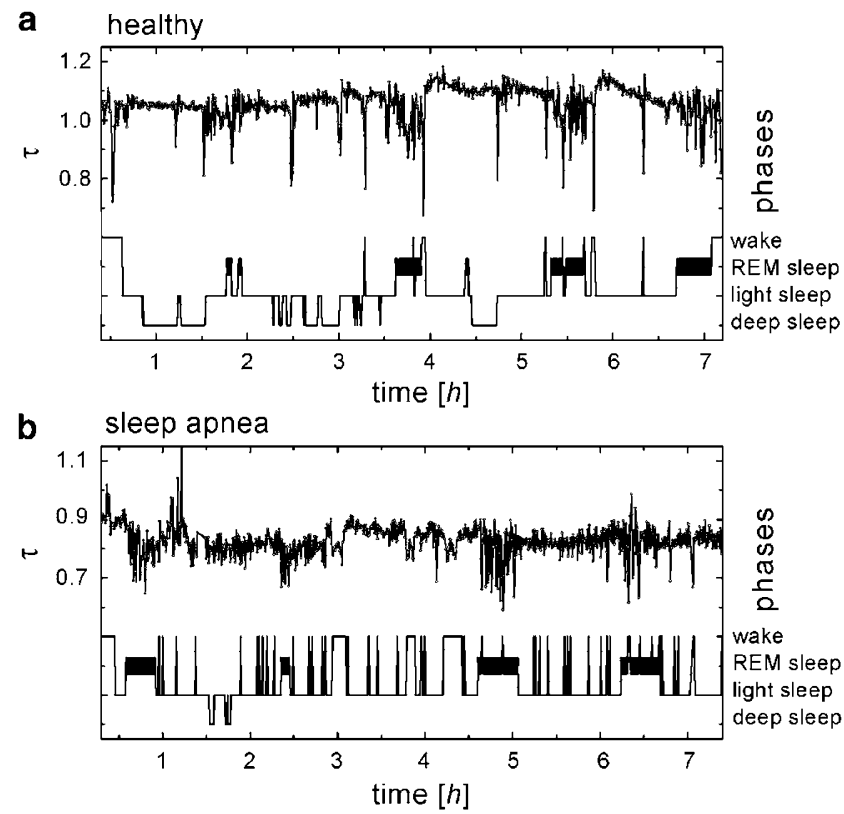

Figure 3 The time course of average interbeat intervals is plotted together with the sleep profile. The recordings present the sleep of (a) a healthy volunteer and (b) a sleep apnea patient. The disturbed sleep can be recognized by the low percentage of deep sleep (short-wave sleep) and the high number of arousal and sleep-wake transitions. wakefulness, REM sleep, light sleep, and deep sleep. In the method, the records are further divided into segments of length $t$, and the variance of the data from polynomial fits in the segments is calculated for different segment sizes $t$. Here, we use the DFA3 method, where cubic polynomials are employed in this detrending procedure. The average variance defines the fluctuation function $F(t)$, which is often found to scale as a power law,

$$
F(t) \sim t^{H} .
$$

In a double-logarithmic plot we can draw $F(t)$ over the time interval $t$ and obtain the exponent $H$ (also denoted by $\alpha$ in some papers) as the slope of the resulting curve. If $H$ has values near 0.5 then we speak of uncorrelated interbeat intervals or short-range correlations. If $F(t)$ shows powerlaw scaling with $H$ being significantly larger than 0.5 we speak of long-range correlations between interbeat intervals.

We found marked differences between deep sleep, light sleep, REM sleep, and intermediate wake states. This finding was consistent over all subjects being investigated. For the healthy subjects (Figure $4 \mathrm{a}$ ), we found almost no long-range correlations during deep sleep $(H=0.52)$, while there are clear long-range correlations during REM sleep $(H=0.78)$, almost similar to wakefulness $(H=0.90)$. Light sleep $(H=0.56)$ was found to be close to deep sleep. The surprising finding of this analysis was that the result remained the same in patients with obstructive sleep apnea (Figure 4b). Again there were almost no long-range correlations in deep sleep $(H=0.54)$ and light sleep $(H=0.43)$ but marked long-range correlations in REM sleep $(H=0.85)$, which were similar to wakefulness $(H=0.90)$.

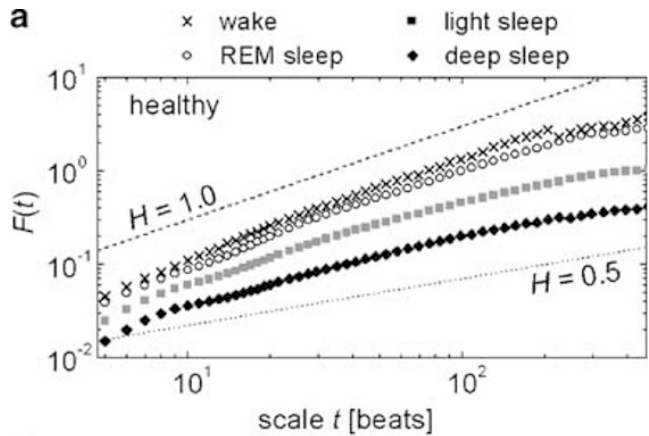

b

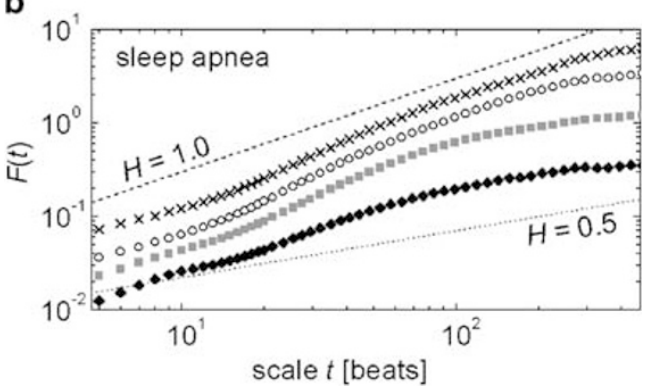

Figure 4 The double-logarithmic plot of the DFA fluctuation functions $F(t)$ over the time scale $t$ allows to determine the scaling behavior and the slopes $H$ during the different sleep stages, deep sleep, light sleep, REM sleep, and the intermediate wake states. (a) The average results for the 14 healthy subjects and (b) The results for the 64 sleep apnea subjects. 
The differences between healthy and sleep apnea subjects were much smaller than the differences between sleep stages. This indicates that the basic mechanisms for heartrate control on an interbeat level did not change very much with sleep apnea. We assume that this basic mechanism is strongly controlled by sleep stages. It seems likely that the long-range correlations during wakefulness and REM sleep are caused by the enhanced influence of the brain on the autonomous nervous system. When this influence is strongly reduced, as is the case during light sleep and deep sleep, the heartbeat intervals behave in a more random fashion.

\section{CONCLUSIONS}

The internal structure of sleep shows clear dynamics that follow a physiological imprinted pattern. This pattern can be described successfully by sleep stages ranging from light sleep to deep sleep and REM sleep. The dynamics of sleep stages can be investigated as such by analyzing the duration of sleep stages in the course of the night. The statistical analysis of sleep-stage durations revealed completely different patterns for the regulation of sleep stages and wakefulness episodes during sleep. This indicates that sleep and wakefulness are not just two parts of a sleep-wakefulness control, but that there exist entirely different mechanisms for their regulation in the brain. This fundamental mechanism is not altered in principle by sleep disorders that have a large impact on sleep fragmentation. Only the parameters of the distributions change.

The analysis of the autonomic nervous system during sleep by the investigation of heart-rate variability gives further insight into the regulation of sleep. We found that when the brain is very active as in the 'dream' - REM stage, heart rate has long-time correlations, like in the wake phase. In contrast, in deep sleep correlations of the heart rate vanish after a small number of beats. In light sleep finally, the heart rate seems to become uncorrelated as well, but only after an increased number of beats. We also compared the altered autonomic nervous system function in obstructive sleep apnea with the results for normal subjects. We found that the differences between the sleep stages are much clearer than the differences between healthy and sleep apnea subjects. This means that the basic heart-rate control in the different sleep stages is very dominant. Obstructive sleep apnea introduces an additional variation on heart rate with a typical bradycardia/tachycardia pattern corresponding to the apnea events, but leaves the basic autonomous nervous system regulation untouched.

Our studies support the view that there is a strong interaction between the central nervous sleep regulation and the autonomous nervous system regulation. Both systems interact and the measurable parameters cannot be interpreted without the knowledge about the current state of the other system.

\section{ACKNOWLEDGEMENTS}

We thank A Bunde, S Havlin, PCh Ivanov, J-H Peter, and HE Stanley for cooperation and useful discussions. JK thanks the German Academic Exchange Service (DAAD) for financial support. We also thank the NIH/National Center for Research Resources (P41 RR13622) for financial support. The healthy volunteers were recorded as part of the SIESTA project funded by the European Union (Biomed-2 BMH-97-2040).

\section{REFERENCES}

Akselrod S, Gordan D, Ubel FA, Shannon DC, Barger AC, Cohen RJ (1981). Power spectrum analysis of heart rate fluctuations: a quantitative probe of beat-to-beat cardiovascular control. Science 213: 220-222.

American Thoracic Society (1989). Indications and standards for cardiopulmonary sleep studies. Am Rev Respir Dis 139: 559568.

Berlad I, Shlitner A, Ben-Haim S, Lavie P (1993). Power spectrum analysis and heart rate variability in stage 4 and REM sleep: evidence for state-specific changes in autonomic dominance. $J$ Sleep Res 2: 88-90.

Bonsignore MR, Romano S, Marrone O, Chiodi M, Bonsignore G (1997). Different heart rate patterns in obstructive apneas during NEM sleep. Sleep 20: 1167-1174.

Bunde A, Havlin S, Kantelhardt JW, Penzel T, Peter JH, Voigt K (2000). Correlated and uncorrelated regions in heart-rate fluctuations during sleep. Phys Rev Lett 85: 3736-3739.

Bunde A, Kropp J, Schellnhuber HJ (eds) (2002). The Science of Disasters-Theories of Disasters in Weather, Biology and Finance. Springer: Berlin.

Carscadon M, Dement WC (2000). Normal human sleep: an overview. In: Kryger MH, Roth T, Dement WC (eds). Principles and Practice of Sleep Medicine, 3rd edn. W.B. Saunders Co.: Philadelphia. pp 15-25.

Grote L, Ploch T, Heitmann J, Knaack L, Penzel T, Peter JH (1999). Sleep-related breathing disorder is an independent risk factor for systemic hypertension. Am J Respir Crit Care Med 160: 18751882.

Hosselet JJ, Norman RG, Ayappa I, Rapoport DM (1998). Detection of flow limitation with a nasal cannula/pressure transducer system. Am J Respir Crit Care Med 157: 1461-1467.

Jenkins C, Davies RJO, Mullins R, Stradling JR (1999). Comparison of therapeutic and subtherapeutic nasal continuous positive airway pressure for obstructive sleep apnoea: a randomized prospective parallel trial. Lancet 353: 2100-2105.

Kantelhardt JW, Koscielny-Bunde E, Rego HHA, Havlin S, Bunde A (2001). Detecting long-range correlations with detrended fluctuation analysis. Physica A 295: 441-454.

Liebovitch LS (1998). Fractals and Chaos Simplified for the Life Sciences. Oxford University Press: New York.

Lo C-C, Nunes Amaral LA, Havlin S, Ivanov PC, Penzel T, Peter JH et al (2002). Dynamics of sleep-wake transitions during sleep. Europhys Lett 57: 625-631.

Penzel T, Conradt R (2000). Computer based sleep recording and analysis. Sleep Med Rev 4: 131-148.

Penzel T, Bunde A, Heitmann J, Kantelhardt JW, Peter JH, Voigt K (1999). Sleepstage-dependent heart rate variability in patients with obstructive sleep apnea. Computers in Cardiology 26: 249252.

Pepperell JCT, Ramdassingh-Dow S, Crosthwaite N, Mullins R, Jenkinson C, Stradling JR et al (2002). Ambulatory blood pressure after therapeutic and subtherapeutic nasal continuous positive airway pressure for obstructive sleep apnoea: a randomized parallel trial. Lancet 359: 204-210.

Roos M, Althaus W, Rhiel C, Penzel T, Peter JH, von Wichert P (1993). Vergleichender Einsatz von MESAM IV und Polysomnographie bei schlafbezogenen Atmungsstörungen. Pneumologie 47: $112-118$. 
Sullivan CE, Issa FG, Berthon-Jones M, Eves L (1981). Reversal of obstructive sleep apnea by continuous positive airway pressure applied through the nares. Lancet 1: 862-865.

Rechtschaffen A, Kales A 1968. A Manual of Standardized Terminology, Techniques, and Scoring System for Sleep Stages of Human Subjects. Public Health Service, US Government Printing Office: Washington, DC.
Sornette D (2000). Critical Phenomena in Natural Sciences. Springer: Berlin. pp 285-320.

Zemaityte D, Varoneckas G, Plauska K, Kaukenas J (1986). Components of the heart rhythm power spectrum in wakefulness and in individual sleep stages. Int J Psychophysiol 4: $129-141$. 